\title{
PRODUÇÃO HIDROPÔNICA DE MORANGUEIRO EM SISTEMA DE COLUNAS VERTICAIS, SOB CULTIVO PROTEGIDO ${ }^{1}$
}

\author{
EUNICE OLIVEIRA CALVETE ${ }^{2}$, ALEXANDRE AUGUSTO NIENOW ${ }^{3}$, CRISTIANE DE LIMA WESP ${ }^{4}$, LUCAS \\ CESTONARO $^{5}$, FRANCIELE MARIANI $^{6}$, IRINEO FIOREZE 7 , DILETA CECCHETTI ${ }^{8}$, TATIANA CASTILHOS ${ }^{9}$
}

RESUMO - O cultivo do morangueiro fora do solo possibilita a eliminação do uso de produtos para desinfecção, reduzindo o consumo de frutos contaminados e a agressão ao meio ambiente, além de proporcionar melhor aproveitamento da área e maior facilidade de manejo da cultura. Este trabalho teve como objetivo avaliar, em ambiente protegido e colunas verticais, dois sistemas de irrigação: gotejamento por estacas (externo) e autocompensante (interno); dois tipos de substratos: Horta 2 e Tabaco 1; com e sem drenagem. A cultivar utilizada foi a Oso Grande. O delineamento foi em blocos casualizados, com os tratamentos dispostos em parcela subsubdividida, com três repetições. Com base nos rendimentos obtidos nos terços superior, médio e inferior das colunas, o sistema de irrigação mais indicado é o gotejamento por estacas (externo), com drenagem na extremidade inferior da coluna. Os substratos não diferem quanto à produção, mas Horta 2 incrementa o teor de antocianina nos frutos.

Termos para indexação: Fragaria ananassa X Duch., cultivo sem solo, nutracêuticos.

\section{HYDROPONIC STRAWBERRY PRODUCTION IN VERTICAL COLUMNS SYSTEM UNDER PROTECTED CULTIVATION}

\begin{abstract}
Since strawberry cultivation in soil less system does not needs disinfection products, it decreases fruits and environmental contamination. Besides it provides a better utilization of the area and makes easy the management of the culture. The objective of this work was to evaluate two irrigation systems: dripping for props (outside) and self compensate (inside); and two types of substrates: Horta 2 and Tabaco 1; with or without draining, on the cultivar Oso Grande of strawberry. The experiment was carried out under protected cultivation and in vertical columns conditions. The experimental design was randomized blocks, with three replications, and the treatments were arranged in a split-plot. The strawberry yield found in the upper, medium, and lower positions of the columns indicates that the dripping for props (outside) is the most efficient irrigation system, since drainage is used in the lower extremity of the column. Although there were no differences substrates, Horta 2 increased the anthocyanins content of the fruits.
\end{abstract}

Index terms: Fragaria ananassa X Duch., soil less cultivation, nutraceutic.

\section{INTRODUÇÃO}

A produção mundial de morangos (Fragaria X ananassa Duch) vem sendo incrementada, variando ao redor de $800.000 \mathrm{t}$, na década de 70, a 3.000.000 t, atualmente. Segundo dados da FAOSTA (2006), no ano agrícola de 2004, os principais países produtores eram os Estados Unidos (1.004.110 t), a Espanha (286.300 t), a Polônia (185.600 t), o Japão (205.000 t) e a Coréia $(210.000 \mathrm{t})$. O Brasil não figura entre os maiores produtores, concentrando sua produção nos Estados de Minas Gerais (41,4 \%), Rio Grande do Sul (25,6 \%), São Paulo (15,4 \%), Paraná (4,7 $\%)$ e Distrito Federal (4\%). Aárea de produção brasileira é estimada em 3.500 ha, sendo a média das propriedades ao redor de 0,5 a 1,0 ha, visando a atender ao mercado in natura e à industrialização
(Pagot \& Hoffmann, 2003).

O cultivo do morangueiro vem crescendo nos últimos anos, acompanhado de problemas, como o uso em larga escala de defensivos agrícolas. Diversas alternativas vêm sendo propostas para minimizar esse inconveniente, destacando-se a técnica de ambiente protegido, que propicia alterações positivas no ambiente de cultivo (Goto \& Tivelli,1998). Entretanto, o morangueiro é facilmente infestado por patógenos de solo, sendo indicada a rotação com outras culturas, prática essa muitas vezes não realizada. Por esta razão, cresceu o emprego de técnicas de desinfestação do solo, como a solarização e a utilização de produtos fumigantes, sendo o brometo de metila o mais amplamente utilizado (Bartual et al., 2002). A proibição do brometo de metila e a preocupação com o consumo de frutos

'(Trabalho 024-07). Recebido em: 18-01-2007. Aceito para publicação em:24-08-2007. Projeto financiado pela FAPERGS (Processo n. ${ }^{\circ} 04 / 0540.3$ PROCOREDES) e a Empresa MEC PRECâ (doação dos substratos).

${ }^{2}$ Profa. de Olericultura -FAMV /UPF, Campus I, Passo Fundo-RS, Cx. P. 611, CEP 99001-970, calveteu@upf.br

${ }^{3}$ Prof. de Fruticultura da FAMV/UPF, Campus I, Passo Fundo-RS, Cx. P. 611, CEP 99001-970, alexandre@upf.br

${ }^{4}$ Acadêmica de Agronomia da FAMV/UPF e Bolsista FAPERGS. cristianewesp@yahoo.com.br

${ }_{5}^{5}$ Acadêmico de Agronomia e estagiário do Setor de Horticultura da FAMV/UPF. lucascestonaro@yahoo.com.br

${ }^{6}$ Acadêmica de Agronomia da FAMV/UPF e Bolsista Probic II/FAPERGS. francielemariani@bol.com.br

${ }^{7}$ Eng.Agr., Especialista, prof. de Solos da FAMV/UPF, labsolos@upf.br

${ }^{8}$ Matemática, Ms.Profa. de Estatística da UPF. cecchetti@upf.br

${ }^{9}$ Farmacêutica, Ms.Profa. Substituta Farmacognosia I e II da UPF. tcastilhos@bol.com.br 
contaminados, além da agressão ao meio ambiente, justificam a necessidade de serem pesquisadas novas alternativas. $\mathrm{O}$ cultivo em sistemas sem solo abre algumas possibilidades, no sentido de combinar ambiente protegido com a eliminação do uso de produtos destinados à desinfecção do solo, além de proporcionar maior produtividade (Fernandes et al., 2002). O mercado atual de morangos apresenta demanda pela produção precoce e fora de safra. Uma das maneiras de conseguir isso é com o cultivo sem solo, pois, através dessa técnica, pode-se antecipar o início da colheita e ainda incrementar o rendimento por área.

No Brasil, embora sejam poucas as pesquisas e pequena a difusão entre os agricultores, segundo Morard (1984), o cultivo em substratos é a técnica de produção sem solo mais empregada em muitos países da Europa, onde o sistema em colunas verticais começou a ser desenvolvido, com o objetivo de melhor aproveitar o espaço físico da área dos ambientes protegidos. Os recipientes de cultivo podem ser compostos de materiais diversos, entretanto os sacos de polietileno apresentam-se como alternativas aos leitos e bancadas (Resh, 1997). Como substratos, podem ser utilizados vários materiais, no entanto são necessários estudos sobre as matérias-primas existentes, assim como as misturas comerciais, relacionando estes com o crescimento dos vegetais (Andriolo et al., 1999).

Na produção de plantas em recipientes, a irrigação e as adubações complementares são essenciais para a manutenção de elevada disponibilidade de água e nutrientes. Como o substrato tem capacidade de armazenamento limitado, a reposição desses elementos deve ser freqüente, de forma a satisfazer as necessidades hídricas e nutricionais das plantas (Bellé,1998). A irrigação por gotejamento destaca-se como a tecnologia de irrigação mais racional, visto ser o método que possibilita maior eficiência no uso da água (Manfrinato apud García et al., 2003).

Os mercados, por sua vez, estão evoluindo e exigem cada vez mais qualidade dos produtos consumidos. Muitos fatores influenciam na qualidade do morango, como a cultivar, as condições ambientais, as práticas culturais agrícolas e as condições de armazenamento. Portanto, embora exista ampla bibliografia sobre a qualidade e o conteúdo nutricional dessa fruta, são necessários constantes estudos que avaliem as alterações na qualidade, em função das cultivares e das práticas de cultivo, entre outros fatores.

Atualmente, há um aumento do consumo de frutas e hortaliças, em decorrência do valor nutritivo e dos efeitos terapêuticos que esses alimentos apresentam por conter diferentes fitoquímicos, muitos dos quais com propriedade antioxidante, que pode estar relacionada com o retardo do envelhecimento e a prevenção de doenças (Henriques et al.,2004). Dentre os compostos fenólicos com propriedade antioxidante, destacam-se os flavonóides que, quimicamente, englobam as antocianinas e os flavonóis, entre outros. As antocianinas são pigmentos solúveis em água, amplamente difundidas no reino vegetal, conferindo várias nuanças de cores entre laranja, vermelho e azul em frutas, flores, folhas e raízes (Francis, apud Lima et al., 2002). Para Cordenunsi et al. (2002), além do tamanho e da forma, a cor é um importante componente na aparência de morangos, definida pelo índice de antocianina.
Este trabalho teve como objetivo avaliar dois sistemas de irrigação e dois tipos de substratos, com e sem o auxílio de drenagem, no cultivo sem solo do morangueiro 'Oso Grande', em colunas verticais sob ambiente protegido.

\section{MATERIAL E MÉTODOS}

O experimento foi realizado no período de julho de 2005 a janeiro de 2006, na Universidade de Passo Fundo, em Passo FundoRS, (latitude de $28^{\circ} 15^{\prime} 39^{\prime \prime} \mathrm{S}$, longitude de $52^{\circ} 24^{\prime} 33^{\prime \prime} \mathrm{O}$, altitude média de $680 \mathrm{~m}$ ), em uma estufa com estrutura de alumínio galvanizado, instalada no sentido nordeste-sudeste, de $430 \mathrm{~m}^{2}$, com teto semicircular, coberta com filme de polietileno de baixa densidade (PEBD), com aditivo antiultravioleta e espessura de $150 \mathrm{~mm}$.

Para o cultivo hidropônico vertical, foram confeccionadas sacolas na forma tubular, com polietileno branco anti UV de 100 $\mathrm{mm}$ de espessura, cortado em segmentos de $2,30 \mathrm{~m}$ de comprimento, formando tubos de $0,39 \mathrm{~m}$ de diâmetro. Esses tubos, com ambas as extremidades amarradas, passaram a ter 2,00 $\mathrm{m}$ de comprimento, distantes $1,50 \mathrm{~m}$ entre si e elevadas $2,10 \mathrm{~m}$ do chão com caibros de eucalipto $(6 \mathrm{~cm} \mathrm{x} 5 \mathrm{~cm})$ e pilares verticais de madeira roliça de eucalipto, com espessura de $30 \mathrm{~cm}$, distantes $3 \mathrm{~m}$ um do outro ao longo da linha. Considerando o eixo dos cilindros, as sacolas de cultivo foram dispostas no sistema de sustentação no espaçamento de $1,5 \mathrm{~m}$ x 1,0 m, ocupando uma área útil de $150 \mathrm{~m}^{2}$ da estufa.

Foram utilizadas mudas da cv. Oso Grande, de aproximadamente $8 \mathrm{~cm}$ de altura, com três a quatro folhas. Antes do transplantio, realizado em 6 de julho de 2005, as sacolas de cultivo foram perfuradas numa inclinação de $45^{\circ}$ para acomodar a muda, com o auxílio de um bastão de metal aquecido.

Os tratamentos (fatorial triplo) foram dispostos em parcelas subsubdivididas, no delineamento de blocos casualizados, com três repetições, sendo testados dois sistemas de irrigação externo (conjunto de irrigação por gotejamento com sistema por estacas) e auto-compensante (conjunto de irrigação por gotejamento interno, constituído de um tubo de $1 / 2$ polegada, com gotejadores a cada $10 \mathrm{~cm}$ ); dois substratos comerciais - Mecplant Horta $2 \AA$ e Mecplant Tabaco $1 \circledast$ (fabricante Wolf Klabin MEC PREC $\AA$, de composição não especificada); sem e com drenagem (camada de $10 \mathrm{~cm}$ de brita na extremidade inferior da coluna).

As irrigações constituíram as parcelas principais, as subparcelas, os substratos e as subsubparcelas a drenagem. Cada parcela foi constituída por 3 colunas com 28 plantas, distribuídas em quatro linhas radiais eqüidistantes. Cada linha envolvia sete plantas verticalmente dispostas e espaçadas $0,25 \mathrm{~m}$ entre si, totalizando 12 colunas por bloco. As colunas foram divididas longitudinalmente em três partes iguais (terço superior, médio e inferior), a fim de estudar a possível ocorrência de um gradiente de produção ao longo das mesmas.

Foram preparadas três soluções nutritivas concentradas, utilizadas conforme o estádio da cultura. As soluções foram elaboradas com base na solução descrita por Fernandes Júnior \& Furlani (2002), porém modificada, sendo compostas da solução A: $1.600 \mathrm{~g}$ de nitrato de cálcio; $120 \mathrm{~g}$ de hidroferro; $6,0 \mathrm{~g}$ de ácido 
bórico; 0,6 $\mathrm{g}$ de sulfato de cobre; 4,0 $\mathrm{g}$ de sulfato de manganês; $2,0 \mathrm{~g}$ de sulfato de zinco e $0,6 \mathrm{~g}$ de molibdato de sódio, dissolvidos em 10 L de água; da solução B: $280 \mathrm{~g}$ de uréia; $300 \mathrm{~g}$ de fosfato monoamônico; $1.000 \mathrm{~g}$ de superfosfato simples; $900 \mathrm{~g}$ de cloreto de potássio e $1.200 \mathrm{~g}$ de sulfato de magnésio, dissolvidos em $10 \mathrm{~L}$ de água; e da solução C: $1.900 \mathrm{~g}$ de superfosfato simples; $420 \mathrm{~g}$ de cloreto de potássio; $100 \mathrm{~g}$ de nitrato de potássio e $1.200 \mathrm{~g}$ de sulfato de magnésio, dissolvidos em $10 \mathrm{~L}$ de água. Até o início da floração, foram utilizadas as soluções A e B, sendo 1,5 L de cada diluídas em $500 \mathrm{~L}$ da solução final. Durante a floração, a fertirrigação foi realizada com as soluções A e C, sendo diluídas da mesma forma anterior. A solução nutritiva foi distribuída nas colunas de cultivo por dois difusores de vazão reguláveis, ajustados para emitir, aproximadamente, $11 \mathrm{~L}$ por hora cada. As soluções nutritivas foram fornecidas às plantas, 2 a 3 vezes por semana.

Foram analisadas as seguintes características físicas nos substratos: densidade, porosidade total, espaço de aeração e capacidade de retenção de água. Para medir a condutividade elétrica (CE), foram, ao acaso, instalados vasos na base das colunas, para coletar a solução nutritiva lixiviada. Para as medidas, foi utilizado um condutivímetro manual.

As variáveis analisadas foram o número e a massa fresca total de frutos por planta, e o número e a massa fresca de frutos comerciais por planta o teor de antocianina presente nos frutos. Determinou-se o teor de antocianina pelo método descrito na Farmacopéia Portuguesa (2002), apud Henriques et al. (2004). Os resultados referentes ao rendimento e teor de antocianina foram submetidos à análise de variância, e as diferenças entre médias comparadas pelo Teste de Tukey, a $5 \%$ de probabilidade.

\section{RESULTADOS E DISCUSSÃO}

A análise das características físicas dos dois substratos estudados demonstrou que a porosidade total do Horta 2 foi de $0,88 \mathrm{~m}^{3} \mathrm{~m}^{-3}$ e do Tabaco 1 de $0,65 \mathrm{~m}^{3} \mathrm{~m}^{-3}$, encontrando-se apenas o Horta 2 dentro da faixa ideal de porosidade ( $>85 \%$ ), segundo De Boodt e Verdonck (1972). Quanto ao espaço de aeração, determinado na tensão de $1 \mathrm{kPa}$, o substrato Horta 2 apresentou valor de $0,18 \mathrm{~m}^{3} \mathrm{~m}^{-3}$ e o Tabaco $1 \mathrm{de} 0,02 \mathrm{~m}^{3} \mathrm{~m}^{-3}$. Segundo os mesmos autores, valores inferiores a $0,10 \mathrm{~m}^{3} \mathrm{~m}^{-3}$ são considerados restritivos ao desenvolvimento das raízes. Os substratos praticamente não diferiram quanto ao armazenamento de água, que foi de $0,29 \mathrm{~m}^{3} \mathrm{~m}^{-3}$ no Horta 2 e de $0,27 \mathrm{~m}^{3} \mathrm{~m}^{-3}$ no Tabaco 1 . De acordo com De Boodt e Verdonck (1972), que caracterizam essa faixa de potencial (de 10 a $50 \mathrm{kPa}$ ) como a fração de água facilmente disponível, é desejável de 20 a $40 \%$ de umidade volumétrica entre esses pontos. Essa faixa é geralmente adotada para definir os limites inferiores de disponibilidade de água (Riviére et al.,1992). Embora os substratos demonstrem algumas diferenças nas características físicas, estas não foram suficientes para impor variações significativas nas variáveis produtivas.

A variação da condutividade elétrica (CE) nos dois sistemas de irrigação e nos dois substratos, ao longo do ciclo da cultura, é apresentada na Figura 1. Na irrigação externa, a CE das soluções lixiviadas nos substratos Horta 2 e Tabaco 1 foi semelhante, em média $0,62 \mathrm{~m} . \mathrm{S} . \mathrm{cm}^{-1}$. Já no sistema de irrigação interna, a CE no substrato Horta 2 apresentou valor mais elevado $\left(1,10 \mathrm{~m} .{\mathrm{S} . \mathrm{cm}^{-1}}^{-1}\right)$ em relação a Tabaco 1 (0,76 m.S.cm $\left.{ }^{-1}\right)$. Moraes \& Furlani (1999) recomendam que a $\mathrm{CE}$ no cultivo de morangueiro, seja mantida entre 1,5 e 2,0 m.S.cm ${ }^{-1}$.

O número total de frutos por planta (Tabela 1), nos diferentes terços da coluna vertical de cultivo, não foi afetado pelo tipo de substrato nem o uso ou não de drenagem na extremidade inferior da coluna. Comparando os sistemas de irrigação, verifica-se que a irrigação externa igualmente não interferiu no número total de frutos produzidos nos diferentes terços da coluna (em média 18,5 frutos por planta), mas a irrigação interna reduziu o número de frutos no terço inferior (14 frutos por planta), diferindo em relação ao terço superior $(18,5$ frutos por planta). $\mathrm{O}$ fato pode ser, possivelmente, atribuído ao maior acúmulo de umidade no terço inferior proporcionado pelo sistema interno de irrigação, em especial quando não utilizada a camada de drenagem, visto que houve uma tendência de redução do número de frutos por planta sem a drenagem, independentemente do sistema de irrigação ou do tipo de substrato. Os tratamentos não influenciaram no número de frutos comerciais produzidos, que foi, em média, de 13,1 frutos por planta, ou seja, em torno de 73,3 $\%$ do total de frutos produzidos.

Quanto ao efeito dos tratamentos sobre a massa fresca de frutos produzidos por planta, diferenças foram verificadas no terço médio da coluna, em que o sistema de irrigação externo proporcionou maior massa total e de frutos comerciais, em relação ao sistema interno (Tabela 2). Apesar de não serem realizadas avaliações quanto ao potencial matricial dos substratos, constatase que a maior massa de frutos foi alcançada no tratamento (irrigação externa) onde os substratos permaneciam mais uniformemente úmidos. No sistema de irrigação externo, cada planta recebe um microgotejador, permitindo melhor distribuição da umidade nas sacolas de cultivo. No sistema de irrigação interno, os gotejadores encontram-se distribuídos linearmente no eixo central da coluna, espaçados a cada $10 \mathrm{~cm}$, ou seja, cada planta não possui o seu gotejador.

$\mathrm{O}$ uso da drenagem incrementou a massa fresca total de frutos apenas no terço inferior da coluna, provavelmente por evitar o excesso de umidade e a concentração elevada de nutrientes neste segmento (Tabela 2), conforme havia sido observado para o número total de frutos por planta (Tabela 1). Também uma tendência de aumento na massa de frutos comerciais no terço inferior foi verificada com a drenagem, contudo não demonstrada estatisticamente.

Os resultados encontrados concordam em parte com Fernandes Júnior \& Furlani (2002), que afirmam a existência de um gradiente decrescente de produção da parte superior para a parte mediana e inferior da coluna, que foi observada pelos autores, possivelmente, em decorrência da não-utilização da drenagem e diferenças quanto ao substrato utilizado, em relação ao presente trabalho.

A produção média por planta, de 187,9 g.planta ${ }^{-1}$ obtida neste trabalho, mostra-se representativa quando relacionada a outros experimentos com sistemas verticais, como os de Fernandes Júnior et al. (2002) e Morard (1984), os quais atingiram, respectivamente, 233,10 e 250 g.planta ${ }^{-1}$, embora tenham realizado 
seus experimentos em outras condições edafo-climáticas e também utilizando outras cultivares. A menor produtividade encontrada em Passo Fundo pode ser explicada pelas baixas temperaturas verificadas ainda no mês de setembro (mínimas de $8^{\circ} \mathrm{C}$ ) e altas no mês de dezembro (máximas de $35^{\circ} \mathrm{C}$ ), prejudicando a polinização em ambos os casos e, conseqüentemente, provocando o abortamento das flores e a produção de frutos. Temperaturas inferiores a $12{ }^{\circ} \mathrm{C}$ e superiores a $25^{\circ} \mathrm{C}$ tornam o pólen inviável, e superiores a $32^{\circ} \mathrm{C}$ produzem abortos florais (Brazanti, 1989).

Os resultados obtidos também demonstraram que o sistema de irrigação externo proporcionou maior massa fresca total de frutos por planta quando associado ao uso de drenagem (221,5 g.planta ${ }^{-1}$ com drenagem e 185,9 g.planta ${ }^{-1}$ sem drenagem). O sistema interno de irrigação não diferiu quanto ao uso ou não de drenagem, produzindo, em média, 182,5 g.planta ${ }^{-1}$. Treder (2006) constatou que o nível de drenagem não foi fator significativo no cultivo de morangueiro sem solo, utilizando irrigação por gotejamento e por capilaridade. $\mathrm{O}$ crescimento e a produção de frutos somente foram influenciados pelas cultivares, entretanto afirma que o maior nível de drenagem $(30 \%)$ ou o menor $(10 \%)$ pode reduzir o número de frutos de maior tamanho (maior que 3 $\mathrm{cm}$ de diâmetro).

Considerando a média geral obtida da massa fresca total de frutos por planta (187,9 g.planta $\left.{ }^{-1}\right)$, na área de $1,5 \mathrm{~m}^{2}$ por coluna, com 28 plantas por coluna, a produtividade estimada do sistema seria de $35.075 \mathrm{~kg} \mathrm{ha}^{-1}$. Ao considerarmos apenas a média da

TABELA 1- Número total e de frutos comerciáveis de morangueiro cv. Oso Grande nos terços superior, médio e inferior de colunas de cultivo vertical, submetidos a dois sistemas de irrigação, dois substratos, com e sem irrigação. Passo Fundo- RS, FAMV/UPF, 2005

\begin{tabular}{|c|c|c|c|c|}
\hline \multirow{3}{*}{ Tratamentos } & \multicolumn{4}{|c|}{ Número total de frutos por planta } \\
\hline & \multicolumn{4}{|c|}{ Posição na coluna (terços) } \\
\hline & Superior & Médio & Inferior & Média \\
\hline Irrigação externa & A $18,8^{\text {ns }}$ & A $18,5^{\text {ns }}$ & A $18,1^{\text {ns }}$ & 18,5 \\
\hline Irrigação interna & A 18,5 & $\mathrm{AB} 17,3$ & B 14,0 & 16,6 \\
\hline Substrato Horta 2 & NS $18,0^{\text {ns }}$ & $17,9^{\mathrm{ns}}$ & $17,0^{\mathrm{ns}}$ & 17,6 \\
\hline Substrato Tabaco 1 & NS 19,4 & 17,9 & 15,0 & 17,4 \\
\hline Com drenagem & NS $18,1^{\text {ns }}$ & $18,6^{\mathrm{ns}}$ & $18,4^{\mathrm{ns}}$ & 18,4 \\
\hline Sem drenagem & Ns 19,2 & 17,2 & 13,7 & 16,7 \\
\hline \multirow[t]{2}{*}{ Média } & 19,7 & 17,9 & 16,0 & \\
\hline & \multicolumn{4}{|c|}{ Número de frutos comerciáveis por planta } \\
\hline Irrigação externa & NS $13,0^{\text {ns }}$ & $14,9^{\mathrm{ns}}$ & $16,8^{\mathrm{ns}}$ & 14,9 \\
\hline Irrigação interna & NS 11,5 & 12,3 & 10,2 & 11,3 \\
\hline Substrato Horta 2 & NS $12,2^{\text {ns }}$ & $12,9^{\mathrm{ns}}$ & $12,1^{\mathrm{ns}}$ & 12,4 \\
\hline Substrato Tabaco 1 & ${ }^{\mathrm{NS}} 12,3$ & 14,2 & 14,9 & 13,8 \\
\hline Com drenagem & NS $12,2^{\text {ns }}$ & $13,8^{\mathrm{ns}}$ & $12,8^{\mathrm{ns}}$ & 12,9 \\
\hline Sem drenagem & ${ }^{N S} 12,3$ & 13,3 & 14,2 & 13,3 \\
\hline Média & 12,3 & 13,6 & 13,5 & \\
\hline
\end{tabular}

Médias antecedidas de letras maiúsculas distintas na linha diferem entre si, , pelo teste de Tukey, a $5 \%$ de significância.

(NS) não-significativo na linha e (ns) na coluna, a $5 \%$ de significância. massa de frutos comerciais $\left(160,8 \mathrm{~g}\right.$ planta $\left.^{-1}\right)$, a produtividade seria de $30.007 \mathrm{~kg} \mathrm{ha}^{-1}$, que pode ser considerada uma boa produtividade. No Rio Grande do Sul, conforme Pagot e Hoffmann (2003), a média de morangos produzidos situa-se próximo de $32.700 \mathrm{~kg} \mathrm{ha}^{-1}$.

O teor de antocianina nos frutos foi influenciado apenas pelo tipo de substrato, independentemente do sistema de irrigação e utilização ou não de drenagem. Os frutos obtidos no substrato Horta 2 apresentaram maior teor de antocianina $(20,93 \mathrm{mg} / 100 \mathrm{~g})$ em relação aos obtidos no substrato Tabaco $1(11,68 \mathrm{mg} / 100 \mathrm{~g})$. Esse acréscimo de 79,2\% na quantidade de antocianinas reflete não somente na maior coloração vermelha dos frutos e, conseqüentemente, na maior atratividade, como também nos benefícios à saúde do consumidor, pela atividade antioxidante. Cordenunsi et al. (2002) avaliaram a composição química e os parâmetros de qualidade dos frutos de 13 cultivares de morangueiro coletados em plantios comerciais no Brasil. O conteúdo de antocianina variou de $13 \mathrm{mg} / 100 \mathrm{~g}$ (cv. Campineiro) a $55 \mathrm{mg} / 100 \mathrm{~g}$ (cv. Mazi). Já Maro et al. (2000), analisando morangos produzidos em Minas Gerais, encontraram valores de $19 \mathrm{mg} / 100 \mathrm{~g}$ (Cv. Guarani) a 13,26 mg/100g (cv. Sweet Charlie). Ressalta-se que, nesses dois trabalhos, os frutos foram produzidos a campo, e não em condições de cultivo sem solo e em ambiente protegido.

TABELA 2 - Massa fresca total e de frutos comerciáveis de morangueiro cv. Oso Grande nos terços superior, médio e inferior de colunas de cultivo vertical, submetidos a dois sistemas de irrigação, dois substratos, com e sem irrigação. Passo FundoRS, FAMV/UPF, 2005

\begin{tabular}{|c|c|c|c|c|}
\hline \multirow{3}{*}{ Tratamentos } & & \multicolumn{3}{|c|}{ Massa fresca total de frutos (g.planta } \\
\hline & \multicolumn{3}{|c|}{ Posição na coluna } & \\
\hline & Superior & Médio & Inferior & Média \\
\hline Irrigação externa & ${ }^{\mathrm{NS}} 200,0^{\mathrm{ns}}$ & $209,9 \mathrm{a}$ & $201,3^{\mathrm{ns}}$ & 203,7 \\
\hline Irrigação interna & ${ }^{\mathrm{NS}} 182,9$ & $176,8 \mathrm{~b}$ & 156,4 & 172,0 \\
\hline Substrato Horta 2 & NS $184,1^{\text {ns }}$ & $189,0^{\mathrm{ns}}$ & $184,0^{\mathrm{ns}}$ & 185,7 \\
\hline Substrato Tabaco 1 & NS 198,8 & 197,7 & 173,7 & 190,1 \\
\hline Com drenagem & NS $191,4^{\text {ns }}$ & $202,0^{\mathrm{ns}}$ & $207,4 \mathrm{a}$ & 200,3 \\
\hline Sem drenagem & ${ }^{\mathrm{NS}} 191,6$ & 184,6 & $150,3 \mathrm{~b}$ & 175,5 \\
\hline \multirow[t]{2}{*}{ Média } & 191,5 & 193,4 & 178,9 & \\
\hline & \multicolumn{4}{|c|}{ Massa fresca de frutos comerciais (g. planta ${ }^{-1}$ ) } \\
\hline Irrigação externa & NS $167,6^{\text {ns }}$ & $182,0 \mathrm{a}$ & $180,1^{\mathrm{ns}}$ & 176,6 \\
\hline Irrigação interna & NS 151,3 & $149,5 \mathrm{~b}$ & 134,0 & 144,9 \\
\hline Substrato Horta 2 & NS 155,2 ns & $161,4^{\mathrm{ns}}$ & $157,2^{\mathrm{ns}}$ & 157,9 \\
\hline Substrato Tabaco 1 & ${ }^{\text {NS }} 163,8$ & 170,0 & 156,9 & 163,6 \\
\hline Com drenagem & NS $160,4^{\text {ns }}$ & $176,6^{\mathrm{ns}}$ & $175,1^{\mathrm{ns}}$ & 170,7 \\
\hline Sem drenagem & ${ }^{\mathrm{NS}} 158,6$ & 154,9 & 139,1 & 150,9 \\
\hline Média & 159,5 & 165,8 & 157,1 & \\
\hline
\end{tabular}

Médias seguidas de letras minúsculas distintas na coluna diferem entre si, pelo teste de Tukey, a $5 \%$ de significância.

(NS) não-significativo na linha e (ns) na coluna, a $5 \%$ de significância. 

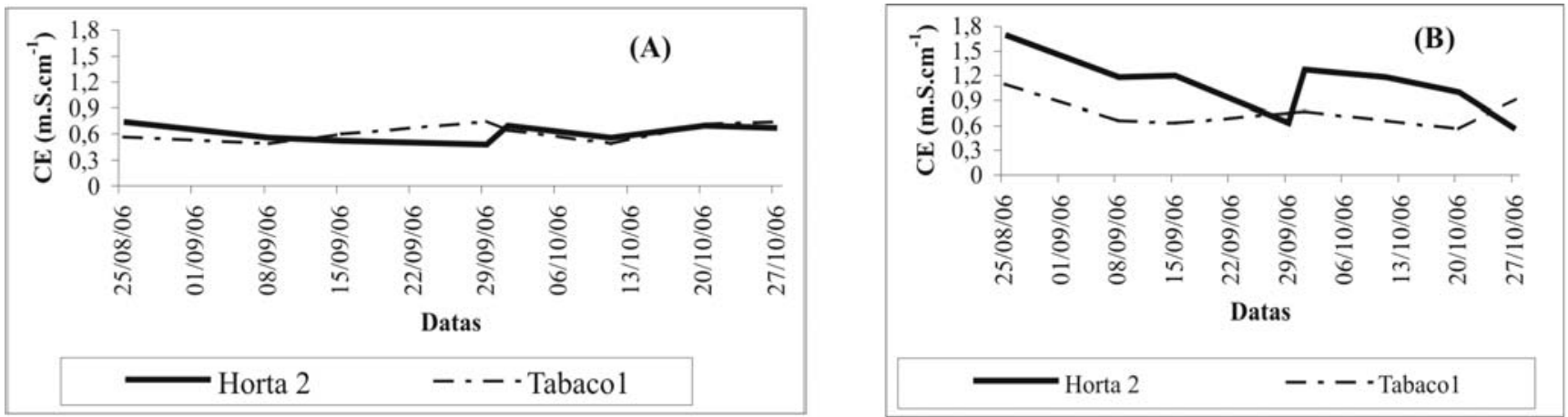

FIGURA 1 - Condutividade elétrica das soluções lixiviadas nos sistemas de irrigação externo (a) e interno (b), nos substratos Horta 2 e Tabaco 1. Passo Fundo-RS, FAMV/UPF, 2005.

\section{CONCLUSÕES}

1-Com base nos rendimentos obtidos nos terços superior, médio e inferior das colunas, o sistema de irrigação mais indicado é o externo, de gotejamento por estacas (aranha), com drenagem na extremidade inferior da coluna.

2-Quanto à produção, os substratos não diferem, mas Horta 2 possibilita maior teor de antocianina.

\section{REFERÊNCIAS}

ANDRIOLO, J.L.; DUARTE, T.S.;LUDKE,L.; SKRESKY, E.C. Caracterização e avaliação de substratos para cultivo de tomateiro fora do solo. Horticultura Brasileira, Brasília, v.17, n.3, p.215219,1999 .

BARTUAL, R.; CEBOLLA, V.; BUSTOS, J.; GINER, A.; LOPEZARANDA, J.M. The spanish project on alternatives to methyl bromide (2): the case of strawberry in the area of Valencia. Acta Horticulturae, Wageningen, v.567, p.431-434, 2002.

BELLÉ, S. Sistemas de irrigação e concentrações de adubação complementar na produção de Gérbera jamesonii cv. 1187 em vaso. 1998. 122f. Tese (Doutorado em Fitotecnia) - Faculdade de Agronomia,Universidade Federal do Rio Grande do Sul, Porto Alegre, 1998.

BRAZANTI E.C. La Fresa. Madri: Mundi-prensa. 1989. 386p.

CORDENUNSI, B. R.; NASCIMENTO, J.R.O. do;GENOVESE, M.I.; LAJOLO, F.M. Influence of cultivar on quality parameters and chemical composition of strawberry fruits grown in Brazil. Journal of Agricultural and Food Chemestry, Washington, v.50, p.2581$2586,2002$.
DE BOOT, M.; VERDONCK, O. The physical properties of the substrates in horticulture. Acta Horticulturae, Wageningen, v.26, p.37-44, 1972

FAOSTA. Produção de morango no mundo em 2004. Disponível em: $<$ http://www.fao.org/es/ess/yearbook $>$. Acesso em :30 jan. 2006.

FERNANDES JÚNIOR, F.; FURLANI, P. R.; RIBEIRO, I. J. A.;CARVALHO, C. R. L. Produção de frutos e estolhos do morangueiro em diferentes sistemas de cultivo em ambiente protegido. Bragantia, Campinas, v. 61, n. 1, p. 25-34, 2002.

HENRIQUES, A.T.; BASSANI, V.L.; RASEIRA, M.do C.; ZUANAZZI,J.A. Antocianos e capacidade antioxidante de frutas. In: SIMPÓSIONACIONAL DO MORANGO, 2., ENCONTRODE PEQUENAS FRUTAS E FRUTAS NATIVAS, 1., 2004, Pelotas. Anais... Pelotas: Embrapa Clima Temperado, 2004. p.271-282. (Documentos, 124)

GARCÍA,C.J.B.; DARLI,A.B.; ANDRADE, A.R. de; OLIVEIRA, M.V.A.M. de; CRUZ, R.L. Irrigação por gotejamento superficial e subsuperficial na cultura de batata com dois sistemas de plantio.Irriga, Botucatu, v.8,n.2, p.150-159,2003.

GOTO, R.; TIVELLI, S. B. Produção de hortaliças em ambientes protegido: condições subtropicais. São Paulo: FUNEP, 1998.319 p.

LIMA, V.L.A.G. de; MÉLO, E.A. de; LIMA, D.E. de S. Fenólicos e carotenóides totais em pitanga. Scientia Agrícola, Piracicaba, v.59, n.3, p.447-450, 2002. 
MARO, L. A. C.; SANTOS,L.O.; MARTINS,R.N.; FERNANDES,M.S.C.D.;CANUTO,R.da Teor de antocianina e flavonóis em morangos cultivados na região norte de Minas Gerais. In: CONGRESSO BRASILEIRO DE FRUTICULTURA, 18. 2004, Florianópolis.Anais..., Florianópolis: Sociedade Brasileira de Fruticultura, 2004. CD-ROM.

MORAES, C.A.G.; FURLANI, P.R. Cultivo de hortaliças de fruto em hidroponia. Informe Agropecuário, Belo Horizonte, v.20, n.200/ 201,p.105-113, 1999.

MORARD, P. Nutrition control of strawberries hydroponically grown in vertical columns using percolate analysis. In: INTERNATIONAL CONGRESS ON SOILLESS CULTURE, 6 ., 1984, Luteren. Proceedings... Luteren: ISOSC, 1984. p. 393-399.
PAGOT, E.; HOFFMANN, A. Produção de pequenas frutas no Brasil. In: SEMINÁRIO BRASILEIRO SOBRE PEQUENAS FRUTAS, 1., 2003, Vacaria. Anais... Bento Gonçalves: Embrapa Uva e Vinho, 2003. p.9-17. (Documentos, 37).

RESCH, H.M. Cultivos hidroponicos: nuevas técnicas de producción. $4^{\text {th }}$ ed. Madrid: Ediciones Mundi-Prensa, 1997. 509p. RIVIÈRE, L.M.; FOUCARD, J.C.; LEMAIRE, F.Irrigation of container crops according to the substrate. Scientia Horticulturae, Piracicaba, n.43, p.339-349, 1990.

TREDER, W. Effect of drainage level on growth and yield of some strawberry varieties cultivated in soilless culture..Disponível em: <http://www.agr.unian.it/ricerca/prog-ric/wg3/neri/treder\%20Lizbona.ntm>. Acesso em: 05 abr. 2006. 